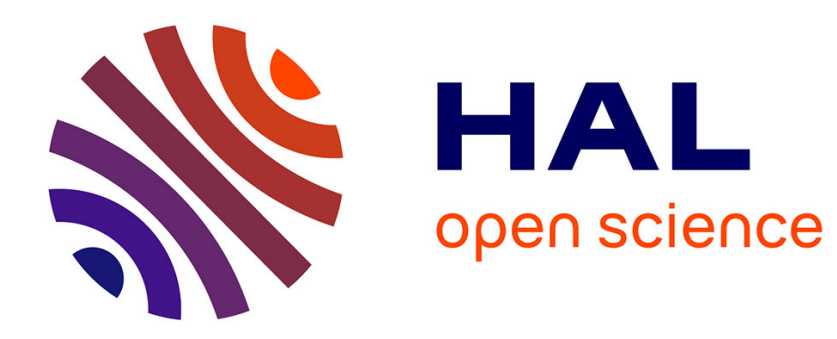

\title{
Itinéraires instables et exposition à la discrimination
} Emmanuelle Marchal

\section{To cite this version:}

Emmanuelle Marchal. Itinéraires instables et exposition à la discrimination. Les Cahiers de la LCD, 2019, 2 (10), pp.23 -42. 10.3917/clcd.010.0023 . halshs-02570651

\section{HAL Id: halshs-02570651 https://shs.hal.science/halshs-02570651}

Submitted on 12 May 2020

HAL is a multi-disciplinary open access archive for the deposit and dissemination of scientific research documents, whether they are published or not. The documents may come from teaching and research institutions in France or abroad, or from public or private research centers.
L'archive ouverte pluridisciplinaire HAL, est destinée au dépôt et à la diffusion de documents scientifiques de niveau recherche, publiés ou non, émanant des établissements d'enseignement et de recherche français ou étrangers, des laboratoires publics ou privés. 


\title{
Itinéraires instables \\ et exposition à la discrimination
}

\author{
EMMANUELle MARChAL
}

Emmanuelle Marchal est sociologue, directrice de recherche CNRS au Centre de Sociologie des Organisations (SciencsPo/CNRS).

emmanuelle.marchal@sciencespo.fr

\section{Introduction}

La discontinuité des trajectoires professionnelles n'est pas sans incidence sur les risques discriminatoires. Elle se traduit en effet par une remise à l'épreuve périodique des individus à la recherche de contrats ou d'emplois stables, qui offre aux employeurs autant d'occasions, ou de tentations, de se saisir de critères discriminatoires pour rejeter leurs candidatures. C'est cette hypothèse que nous cherchons à creuser dans cet article, en suivant le fil des liens entre précarité des emplois et discrimination à l'embauche. À cette fin, nous prenons appui sur le traitement des plaintes déposées au Défenseur des droits entre 2013 et 2015 en nous intéressant spécifiquement à celles qui débouchent sur la mise en cause d'employeurs (voir méthodologie en annexe) : $21 \%$ de ces plaintes concernent précisément des personnes dont le contrat n'est pas renouvelé ou qui ne parviennent pas à obtenir un emploi stable chez l'employeur privé ou public chez qui ils travaillent pour des durées limitées (Marchal, 2018). Ils y sont intérimaires, salariés à durée déterminée, bénéficiaires de contrats 
d'usage $^{18}$ ou simplement mis et remis à l'essai, sans parvenir à se stabiliser durablement. La survenue d'un évènement dans la trajectoire de ces individus, une grossesse, un accident du travail, un problème de handicap, le simple vieillissement de la personne ou son origine étrangère semblent être la cause de leur non-réemploi, du refus de les titulariser ou de leur accorder un emploi stable. Ces situations ne manquent pas d'éveiller les soupçons des juristes du Défenseur qui traitent de telles plaintes: pourquoi la compétence d'une personne qu'un employeur réembauche périodiquement est-elle soudainement remise en cause?

De tels risques discriminatoires sont mal saisis dans les travaux existants pour des raisons importantes à mettre en évidence. En effet, la discrimination à l'embauche est principalement évaluée à l'aide d'expérimentations, de testings ${ }^{19}$, centrés sur certains critères (le nom, le prénom ou la nationalité du candidat pour saisir son origine, le lieu de résidence, parfois le sexe ou l'âge ou la religion). Ce que l'on sait des offres auxquelles postulent les candidats réels ou fictifs reste très succinct $^{20}$. On connaît le titre de l'emploi testé, sans pouvoir prendre en compte les qualités de l'emploi ou des contrats proposés. Or la stabilisation des parcours professionnels constitue un enjeu crucial dans la période contemporaine. La part des embauches en CDD ne cesse d'augmenter pour représenter $87 \%$ des flux en 2017 (Milin, 2018). Multipliées par 2,5 depuis 2001, les entrées en CDD se font par ailleurs sur des contrats de plus en plus courts : $83 \%$ d'entre eux

\footnotetext{
${ }^{18}$ Les contrats d'usage sont des contrats temporaires dont l'usage n'est autorisé que dans des secteurs d'activités précis (tourisme, hôtellerie restauration, spectacle, audiovisuel, déménagement...). Étant source de souplesse pour l'employeur et de précarité pour le salarié, le recours aux CDD d'usage est strictement encadré.

${ }^{19}$ Les testings consistent à envoyer deux à deux des candidatures fictives à de réelles offres d'emploi. Ces candidatures (CV et lettres de motivation) sont comparables au regard de toutes les caractéristiques sauf une, qui représente la variable à tester (sexe, âge, nom à consonance maghrébine du candidat...). La comparaison des chances d'accéder à l'étape suivante du recrutement est utilisée pour donner la mesure de la discrimination.

${ }^{20}$ A fortiori lorsque les analyses sont centrées sur le « ressenti » des discriminations dans l'emploi, on sait très peu de chose sur la situation incriminée et ne disposons d'aucune donnée sur les emplois auxquels les individus cherchent à accéder.
} 
durent moins d'un mois et certains ne couvrent qu'une seule journée. Le raccourcissement de la durée des contrats affecte également les missions d'intérim. Et de fait, la probabilité qu'un salarié en CDD ou qu'un intérimaire occupe un emploi stable après ce type de contrat, ne cesse de diminuer (Picart, 2014). Cependant, cette probabilité varie avec les caractéristiques sociodémographiques des candidats: les descendants d'immigrés sont surexposés à la précarité et à l'instabilité des emplois (Cusser et al., 2015). Dès le début de leur vie active, ceux qui sont d'origine africaine passent plus souvent par des périodes de chômages, par des contrats courts et des missions d'intérim. Les inégalités d'accès à l'emploi stable et à temps plein frappent tout particulièrement les femmes d'ascendance migratoire (Bruneau et al., 2016). On peut donc s'attendre à une augmentation des discriminations selon l'origine lorsqu'est mise en jeu la qualité des emplois $^{21}$.

L'augmentation des contrats courts s'accompagne de celle des réembauches chez le même employeur (Benghalem, 2016). Celles-ci concernent des intérimaires et des intermittents du spectacle, bénéficiaires de contrats d'usage, mais pas seulement. La pratique des réembauches s'accroît et peut prendre place chez le dernier employeur ou chez un ancien employeur avec une interruption entre deux périodes d'emploi. De telles précisions ne sont pas sans incidences sur notre sujet. Elles redessinent la figure type du candidat discriminé, qui n'est plus nécessairement cet inconnu que l'employeur rejette sans prendre en compte ses caractéristiques productives ${ }^{22}$, mais quelqu'un qu'il connaît et qu'il a vu travailler. Quelque chose se produit après la première embauche, qui amène l'employeur à réviser son jugement. C'est pourquoi on s'attend à ce que d'autres critères discriminatoires soient impliqués dans ces non-renouvellements, comme la santé ou le

\footnotetext{
${ }^{21}$ On remarque à l'inverse, que l'accès à des emplois peu qualifiés de courte durée peut faire l'objet de "discrimination positive » en faveur de la main-d'œuvre immigrée (Meziani, 2014 ; Remichi-Meziani, 2015).

${ }^{22}$ Cette représentation est très présente dans l'organisation des testings et dans la littérature économique.
} 
handicap. Les travaux montrent en ce sens, que les trajectoires instables ne sont pas sans impact sur la santé (Moulin et al., 2009 ; Fontaine et Malherbet, 2016). Les accidents du travail, en particulier sont plus nombreux chez les salariés en contrats à durée déterminée ou temporaire. Il se peut que l'espoir d'être réembauché amène l'individu à faire plus d'efforts, mais aussi à prendre plus de risques qui ont des incidences sur sa santé.

Ces travaux forment la toile de fond des hypothèses que nous travaillons dans cet article, où nous analysons la texture des liens entre risques discriminatoires et instabilité des parcours professionnels. Nous montrons d'abord, à partir d'une étude de cas approfondie, que les renouvellements de contrats s'accompagnent de remises en cause permanentes des compétences des individus. Nous verrons ensuite, à partir de divers exemples, que la recherche d'un emploi stable là où l'on a déjà fait ses preuves; ne va pas de soi, et les raisons pour lesquelles les obstacles rencontrés peuvent éveiller le sentiment d'être discriminé. Nous montrerons enfin ce qui contribue à affaiblir la présomption de discrimination, en nous attardant sur l'exemple des contrats d'usage.

\section{Discontinuité des périodes d'emploi et fréquence des remises à l'épreuve}

L'enchaînement de contrats de courte durée, surtout lorsque s'intercalent des périodes sans emploi, se traduit par des mises à l'épreuve périodiques des salariés. Ce phénomène se renforce lorsque le salarié est affecté dans des lieux de travail différents suivant les contrats. C'est le cas du personnel qui effectue des remplacements dans la fonction publique dont il va être question ici (Bresson, 2016). Nous proposons de rentrer dans l'histoire d'un cas précis, celui de M. T. (réclamation $n^{\circ} 16$ ) qui a déposé une plainte au Défenseur début 2013, pour discrimination à l'égard de la santé, du handicap et de l'âge. Nous pourrons ainsi comprendre de quelle manière la 
multiplicité des contrats et des évaluations peut augmenter les risques discriminatoires.

M. T. est employé comme « agent d'entretien et d'hygiène » dans des établissements scolaires où il fait des remplacements de titulaires qui s'absentent ponctuellement pour diverses raisons (congés, maternité, maladies, accidents du travail). Lui-même est contractuel mais employé dans des conditions précaires, juridiquement floues (Gaboriau, 2019), et affecté dans des lycées professionnels au gré des besoins. En récapitulant son itinéraire professionnel à partir des contrats fournis dans son dossier, on s'aperçoit qu'il en a cumulé 19 sur une durée de 3 ans et demi.

Tableau

Une succession de 19 contrats

\begin{tabular}{|c|c|c|c|c|}
\hline $\begin{array}{l}\text { Période du } \\
\text { contrat }\end{array}$ & $\begin{array}{l}\text { Durée des } \\
\text { contrats } \\
\text { en jours }\end{array}$ & $\begin{array}{l}\text { Durée des } \\
\text { périodes } \\
\text { d'essai }\end{array}$ & $\begin{array}{l}\text { Temps } \\
\text { de } \\
\text { travail }\end{array}$ & $\begin{array}{l}\text { Lieux } \\
\text { d'affectation }\end{array}$ \\
\hline 9/3/09 au 3/4/09 & 26 & 8 & complet & Lycée A \\
\hline $\begin{array}{l}4 / 4 / 09 \\
31 / 5 / 09\end{array}$ & 57 & & complet & Lycée A \\
\hline $\begin{array}{ll}1 / 6 / 09 & \text { au } \\
10 / 7 / 09 & \end{array}$ & 40 & & complet & Lycée A \\
\hline $1 / 9 / 09$ au 4/9/09 & 4 & 8 & complet & Lycée B \\
\hline $\begin{array}{l}5 / 9 / 09 \\
31 / 12 / 09\end{array}$ & 138 & 8 & complet & Lycée C \\
\hline $\begin{array}{ll}1 / 1 / 10 & \text { au } \\
30 / 4 / 10 & \end{array}$ & 120 & 8 & complet & Lycée D \\
\hline $\begin{array}{l}1 / 9 / 10 \\
30 / 9 / 10\end{array}$ & 30 & 8 & complet & Lycée E \\
\hline $\begin{array}{ll}1 / 10 / 10 & \text { au } \\
31 / 12 / 10 & \\
\end{array}$ & 92 & & complet & Lycée E \\
\hline $\begin{array}{ll}1 / 1 / 11 & \text { au } \\
28 / 2 / 11 & \end{array}$ & 59 & & complet & Lycée E \\
\hline
\end{tabular}




\begin{tabular}{|c|c|c|c|c|}
\hline $\begin{array}{ll}1 / 3 / 11 & \text { au } \\
30 / 4 / 11 & \end{array}$ & 61 & & complet & Lycée E \\
\hline 1/5/11 au 8/7/11 & 69 & 8 & complet & Lycée F \\
\hline $\begin{array}{ll}30 / 1 / 12 & \text { au } \\
12 / 2 / 12 & \end{array}$ & 14 & 8 & $50 \%$ & Lycée B \\
\hline $1 / 3 / 12$ au $8 / 4 / 12$ & 39 & 8 & complet & Lycée E \\
\hline $\begin{array}{ll}17 / 9 / 12 & \text { au } \\
3 / 10 / 12 & \end{array}$ & 17 & 8 & $50 \%$ & Lycée G \\
\hline $\begin{array}{ll}04 / 10 / 12 & \text { au } \\
9 / 10 / 12 & \end{array}$ & 6 & & $50 \%$ & Lycée $\mathrm{G}$ \\
\hline $\begin{array}{ll}17 / 9 / 12 & \text { au } \\
14 / 10 / 12 & \end{array}$ & 21 & 8 & $50 \%$ & Lycée $\mathrm{G}$ \\
\hline $\begin{array}{ll}10 / 10 / 12 & \mathrm{au} \\
22 / 10 / 12 & \\
\end{array}$ & 12 & & $50 \%$ & Lycée $\mathrm{G}$ \\
\hline $\begin{array}{ll}12 / 11 / 12 & \text { au } \\
2 / 12 / 12 & \end{array}$ & 21 & 8 & $50 \%$ & Lycée $\mathrm{G}$ \\
\hline $\begin{array}{ll}15 / 10 / 12 & \mathrm{au} \\
28 / 10 / 12 & \end{array}$ & 14 & 8 & $50 \%$ & Lycée H \\
\hline Soit 19 contrats & 840 jours & 96 jours & & 8 lycées \\
\hline
\end{tabular}

La plupart sont à plein temps, mais un accident du travail survenu au bout de 3 ans lui vaut d'obtenir une reconnaissance de la qualité de travailleur handicapé (RQTH), et de travailler par la suite à mi-temps en étant dispensé de certaines tâches. Quelques mois plus tard, il reçoit une lettre de la part de son employeur, lui indiquant qu'on ne lui confiera plus de mission, comme on peut le lire ici.

«J'ai eu connaissance de l'évaluation établie par le lycée G. Cet établissement fait part d'un certain nombre de réserves quant à votre manière de servir. Il apparaît un relâchement, dans la qualité du travail rendu, ainsi qu'un défaut de respect des consignes données par votre hiérarchie. Des problèmes d'autonomie ainsi qu'un manque d'investissement ont été relevés. Au regard de ces éléments je vous 
informe que la région R. ne vous confiera plus d'autres missions de remplacement ». (Extrait du courrier de l'employeur à M. T.).

Pour M. T., il ne fait aucun doute qu'il s'agit là d'une décision à caractère discriminatoire, en lien avec son état de santé et son âge. Il a 52 ans au moment des faits et estime qu'il n'a pratiquement aucune chance de retrouver un travail. Il s'adresse d'abord au syndicat avant de se tourner vers le Défenseur des droits à qui il relate les propos tenus par la directrice du recrutement, qui aurait fait état de la difficulté de le placer à son âge avec son handicap. Précisons d'emblée qu'il n'obtiendra pas gain de cause. Deux choses jouent en effet contre lui : on lui a confié de nouvelles missions après sa déclaration RQTH, ce qui tend à montrer que ses problèmes de santé et son handicap n'ont pas fait obstacle à son réemploi. D'autre part, l'évaluation de son travail par le lycée G. est particulièrement négative. Le plaignant ne le nie pas. Mais il attribue celle-ci à la médisance des agents titulaires hostiles à ce qu'on remplace «une RQTH par un RQTH», probablement parce que des tâches supplémentaires leur incombent dans ces situations.

L'épais dossier envoyé au Défenseur par le plaignant saisit le lecteur, non seulement à cause du nombre de contrats insérés, mais aussi parce que les lycées dans lesquels il est affecté changent à plusieurs reprises. M. T. est envoyé dans une dizaine d'endroits différents durant ces 3 années. Certains lycées sont loin de chez lui, comme il prend soin de le préciser sur une feuille du dossier : celui qui a fourni la mauvaise évaluation est justement à une heure de son domicile. Or il y travaille à mi-temps. D'autre part, un autre lycée qui l'emploie au même moment, pour l'autre partie de son mi-temps, fournit une évaluation positive de son travail. Certes il n'y a passé que 15 jours alors qu'il a été employé durant 2 mois et demi dans l'autre. Certaines évaluations auraient-elles plus de poids que d'autres? Leur hétérogénéité laisse entrevoir que son travail peut être apprécié ici et non ailleurs. La dispersion des jugements, ainsi que leur diversité, permettent à l'employeur de s'en tenir aux plus défavorables, envoyés au 
Défenseur pour souligner qu'il ne s'agit pas d'une nouveauté : il lui a été signifié à deux reprises que sa «manière de servir » a laissé à désirer dans tel lycée.

Cette mémoire des évaluations passées ne joue pas en revanche au moment de la signature des contrats, qui semble permettre leur oubli. Chaque nouvelle affectation se traduit, en effet, par le démarrage d'une nouvelle période d'essai de 8 jours. Certes, celle-ci n'est pas longue. Mais au regard de certains contrats qui ne durent que 15 jours, cela peut représenter la moitié du temps de travail. Et l'on peut observer, que même lorsqu'il est affecté deux fois dans le même lycée, pour peu qu'il ait travaillé ailleurs entre-temps, M. T. est à nouveau remis à l'essai. Les périodes d'essai représentent ainsi plus de $10 \%$ de son temps de travail. Comme nous l'avons vu, l'insécurité des parcours professionnels est corrélée à une augmentation des problèmes de santé (Moulin et al., 2009; Fontaine et Malherbet, 2016). M. T. se trouve précisément arrêté pour « accident de travail » lorsque le chef d'établissement du lycée G. rédige son évaluation. N'est-ce pas là une raison supplémentaire de sa connotation très négative?

\section{Diversité des obstacles à l'obtention d'emplois pérennes}

L'instabilité des itinéraires suscite de fortes attentes à l'égard de contrats de longue durée et de statuts protecteurs. Mais les obstacles à la recherche d'emplois pérennes surgissent là où on ne les attend pas : grossesse, état de santé, handicap, origine, apparaissent alors aux plaignants comme la raison évidente de leur rejet.

C'est le cas de $\mathrm{M}^{\text {me }} \mathrm{G}$., qui est employée en tant que maître-nageuse depuis 18 mois par une grande collectivité territoriale et fait des remplacements au gré des besoins dans plusieurs piscines (réclamation $\mathrm{n}^{\circ} 29$ ). Travaillant sur la base de CDD mensuels renouvelés en continu, elle postule pour obtenir un CDD d'un an dans cette même 
collectivité pour exercer le même emploi. Un tel précédent ne lui confère "aucune priorité pour obtenir le poste visé », comme le précise le jugement du tribunal administratif où elle dépose un recours pour discrimination à l'embauche, parallèlement à sa plainte au Défenseur des droits. En effet, elle est enceinte au moment des faits et reste persuadée que cette grossesse a joué en sa défaveur. Il y avait cinq prétendants à l'emploi pour l'offre de quatre CDD durables, et ce sont quatre hommes qui ont été recrutés, tandis qu'elle est la seule à n'avoir rien obtenu. Et pourtant, elle était la seule en fonction à se présenter. L'évaluation du jury reste souveraine en la circonstance. Elle est formulée de la manière suivante : «Malgré son expérience au sein des piscines communautaires et son parcours sportif, $M^{\text {me }}$ G. n'a pas su convaincre le jury de sa motivation. Son manque de dynamisme ressenti lors de l'entretien n'a pas permis de retenir sa candidature ».

Ainsi, bien que $\mathrm{M}^{\mathrm{me}} \mathrm{G}$. soit reconnue comme compétente pour exercer des emplois de courte durée, elle ne le semble plus tout à fait lorsqu'il s'agit d'obtenir un contrat de plus longue durée. Cet argument revient fréquemment dans les réponses des employeurs interpellés, qui considèrent qu'il est légitime de moduler leur niveau d'exigences en fonction de la durée des contrats proposés. La capacité à s'intégrer à des équipes ou à l'entreprise, de prendre des initiatives ou de se faire force de proposition, prendrait ainsi le pas sur les critères d'opérationnalité lorsqu'il s'agit de pérenniser un contrat.

Les enjeux s'accroissent encore davantage lorsqu'il s'agit d'intégrer la fonction publique pour obtenir le statut de fonctionnaire. Deux critères, en particulier, sont sensibles en ces occasions, comme le montre l'analyse des plaintes déposées au Défenseur: la santé et le handicap. Dans l'exemple donné par cette juriste, on s'offusque d'une déclaration d'inaptitude à l'encontre d'un militaire qui a pourtant largement fait ses preuves.

«Quand vous avez des contractuels qui servent, par exemple, sous contrat pendant 6 ans, des militaires sous contrat, et qu'ils servent parfaitement, et 
qui déclarent avoir eu une sclérose en plaques il y a 12 ans. Arrive le moment de la titularisation et on dit : vous êtes inapte parce qu'il y a 12 ans, vous avez eu une sclérose en plaques. Eh bien, là, on considérera que c'est une discrimination (...). Puisqu'il a été très bien noté, et puis, qu'il a réussi, en plus, les épreuves d'aptitude physique et sportive au moment de la titularisation ». (Entretien, juriste du pôle public).

Si le critère du handicap est lui aussi sensible dans la fonction publique, c'est parce que la loi du 11 février 2005 ouvre la possibilité de recruter des personnes handicapées sur des emplois de contractuels, qui peuvent demander à être titularisées ensuite, au bout d'un an ou deux. Les refus de titularisation font périodiquement l'objet de réclamations auprès du Défenseur de la part des contractuels embauchés dans ce cadre. Ils estiment qu'ils n'ont pas obtenu les aménagements nécessaires à la démonstration de leurs compétences, et qu'ils ne sont pas jugés convenablement pour cette raison. La question des «aménagements raisonnables» est au centre des débats et l'équilibre recherché semble difficile à trouver ${ }^{23}$.

Par-delà la question de la pérennité des emplois, se joue aussi celle du statut auquel il est permis d'accéder, avec les avantages y afférant. Nous pensons, ici, aux bénéfices du statut des cheminots de la SNCF, dont ont été écartés les salariés marocains au moment de leur embauche. Au terme d'une action collective et d'une affaire judiciaire qui aura duré près de $14 \mathrm{ans}$, l'employeur s'est vu finalement condamner pour discrimination en raison de l'origine et de la nationalité (Chappe et Keyani, 2018).

\footnotetext{
${ }^{23}$ Il peut s'agir d'adapter des machines, des outillages ou des postes de travail, ou encore d'aménager l'accès au lieu de travail, etc. Les employeurs sont tenus par la loi de prendre les mesures «appropriées » qui vont permettre aux travailleurs handicapés d'accéder à l'emploi correspondant à leur qualification et d'y progresser « sous réserve que les charges consécutives à la mise en œuvre de ces mesures ne soient pas disproportionnées, compte tenu des aides qui peuvent compenser en tout ou partie les dépenses supportées à ce titre par l'employeur» (L. n 2005-102, 11 févr. 2005, pour l'égalité des droits et des chances, la participation et la citoyenneté des personnes handicapées, art. 24).
} 
Dans sa réclamation, $\mathrm{M}^{\mathrm{me}} \mathrm{D}$. considère, elle aussi, qu'il est pénalisant d'être contractuelle, même en CDI, plutôt que fonctionnaire (réclamation $\mathrm{n}^{\circ} 30$ ). Dotée d'un diplôme d'aide médicopsychologique, elle exerce dans une maison de retraite où elle est appréciée. Embauchée à partir de février 2013, au départ pour un mois, son contrat est renouvelé à quatre reprises jusqu'en décembre 2014. Apprenant qu'un concours sur titre est ouvert dès 2013, elle présente sa candidature mais n'est pas retenue. Les explications fournies oralement par la directrice seraient les suivantes : étant originaire de la Réunion, sa titularisation devrait s'accompagner de congés bonifiés, ce qui coûterait trop cher à la structure. Tenant néanmoins à garder la salariée, la directrice lui propose un CDI. La réclamante comprend plus tard ce que cela signifie, en voyant ses collègues titulaires bénéficier de primes et de droits qui lui sont refusés. «C'est alors que je me suis sentie abusée et j'ai réellement compris ce qu'était un CDI dans la fonction publique » précise-t-elle.

Sa déception est telle qu'elle cherche un nouvel emploi pour manifester son mécontentement et trouve rapidement un CDI dans une autre maison de retraite. Juste avant de signer, elle demande un ultime entretien avec son ancienne directrice, à qui elle veut donner sa démission en main propre. Elle apprend ensuite que son nouveau contrat a été attribué à quelqu'un d'autre en urgence. La correspondance se termine de la façon suivante :

«Je ne voudrais pas faire ma propre conclusion, mais $\mathrm{j}$ 'ai appris que la directrice et la cadre de santé de l'autre maison de retraite se connaissaient bien. Je suis donc sans emploi, ainsi que mon mari. Pas d'indemnité à Pôle emploi car il y a eu démission, et mes démarches pour retrouver un autre emploi sont très difficiles car il faut justifier ma démission ». (Réclamation $\mathrm{n}^{\circ} 30$ ).

Dans cette affaire, le Défenseur ne parvient pas à établir l'existence d'une discrimination. La plainte est affaiblie par le fait que le jury ne 
fait pas d'allusion à ses origines. Par ailleurs, elle n'a que 18 mois d'ancienneté au moment du concours, et ne s'est pas présentée à un deuxième concours qui aurait pu constituer une nouvelle opportunité pour sa titularisation.

Ces exemples dans le secteur public ne doivent pas donner à penser qu'il est plus souvent le siège de discriminations que le secteur privé. Rappelons à ce sujet l'affaire qui a opposé un groupe de l'aéronautique à $\mathrm{M}$. X, dont le nom signalait l'origine étrangère (CA Toulouse, 19 févr. 2010, n 08/06630). La candidature de M. X a été rejetée au profit d'un candidat M. Y, d'origine hexagonale, pour accéder à un poste d'affûteur commande numérique en CDI. Tous deux étaient intérimaires et donc déjà bien connus, mais $\mathrm{M}$. X avait plus d'ancienneté et deux contrats à son actif sur deux postes différents dans cette même entreprise. M. Y avait moins d'expérience mais un diplôme de niveau supérieur à l'autre, ce qui justifiait la préférence accordée par l'entreprise. Dans cette affaire, le jugement invalide le raisonnement, car plusieurs personnes occupant le même poste ont le même niveau de diplôme que M.X, attestant qu'il est suffisant. La contribution de la Halde (autorité qui précède le Défenseur des droits) est décisive dans ce dossier : ses investigations montrent que sur les 288 personnes recrutées sur le même site entre 2000 et 2006, seules deux recrues ont un nom de famille d'origine maghrébine semblable à M. X.

Ainsi donc, les exigences que rajoutent les employeurs privés ou publics à l'occasion de la pérennisation des contrats, peuvent-elles être jugées comme empreintes de critères discriminatoires, alors même qu'elles semblent uniquement motivées par des critères de compétences. 


\section{Brouillage des pistes et affaiblissements des plaintes}

De telles affaires où la discrimination est établie restent rares. Nous avons vu à plusieurs reprises que les réclamants se trouvaient en situation de faiblesse pour accréditer leur plainte et obtenir gain de cause. Il y a à cela des raisons plus ou moins objectives, mais aussi des considérations juridiques qui tiennent à la difficulté de qualifier la situation dans laquelle se trouve une personne dont le contrat n'est pas renouvelé. Les décisions de non-renouvellement des contrats ne sont pas soumises à l'obligation d'une communication, ni même d'une motivation. Elles ne peuvent être assimilées ni à des licenciements ni à des démissions (Gaboriau, 2009).

Lorsqu'une plainte donne lieu à instruction dans le secteur public, l'argument qui revient le plus souvent dans la bouche de l'employeur est «l'intérêt du service ». Cette notion recouvre une large gamme de situations : raisons budgétaires, réorganisations, suppressions de poste, exigence de continuité du service rendu. L'intérêt du service représente un « sésame » derrière lequel s'abrite l'administration pour se justifier. «C'est un motif fréquent pour légitimer des changements de postes, ou des non-renouvellements de contrats. Dans ce cas, la situation du requérant est d'autant plus fragile qu'il ne dispose pas de droit à faire valoir. Qu'il s'agisse de titularisation, de renouvellement de contrat, l'administration dispose en effet d'un pouvoir discrétionnaire sur lequel le juge n'exerce qu'un contrôle minimum. » (Dumortier, 2016, p. 355).

La situation des plaignants n'est pas plus enviable dans le secteur privé. L'analyse de la jurisprudence des cours d'appel montre, en effet, que le non-renouvellement de contrats fait l'objet de nombreuses plaintes pour discrimination devant les juridictions, mais aussi que l'apport de preuves est particulièrement difficile dans cette situation (Bossu, 2014). À titre d'exemple, on citera le cas d'un intérimaire, qui après 15 ans de travail dans la même entreprise et plus de 80 contrats de travail temporaire n'obtient pas la requalification de son contrat en 
CDI, car il n'apporte pas la preuve qu'il s'est effectivement porté candidat pour cela. Le statut du plaignant reste difficile à saisir lorsque les contrats courts s'enchaînent sous la forme de contrats d'usage et qu'ils représentent une règle dans les relations d'emploi (voir encadré).

\section{Embauche, carrière ou licenciement \\ De quoi parle-t-on?}

Dans la réclamation ( $\mathrm{n}^{\circ}$ 5) de $\mathrm{M}^{\text {me }}$ B., on note le flottement permanent des termes mobilisés pour qualifier la situation. $\mathrm{M}^{\mathrm{me}} \mathrm{B}$. a travaillé pendant 37 ans dans des entreprises du secteur de la presse où elle a enchaîné des contrats. Les 6 dernières années se passent chez le même employeur où elle bénéficie de quelque 150 contrats d'usage en tant que rédactrice conceptrice. Le syndicat qui gère son placement lui indique du jour au lendemain qu'elle ne peut être gardée en raison de difficultés rencontrées dans le secteur. Elle a 56 ans au moment des faits et s'estime discriminée en raison de son âge. Pour donner du poids à sa réclamation, elle se munit de nombreux témoignages attestant que sa compétence n'est pas en cause.

Ce qui nous intéresse ici, c'est de rendre compte des hésitations qui entourent la qualification des faits. Doit-on considérer que la situation incriminée touche à l'embauche, la carrière ou le licenciement?

$\mathrm{Au}$ départ $\mathrm{M}^{\mathrm{me}} \mathrm{B}$. prend la troisième option en réclamant de savoir pourquoi on l'a « mise à la porte sans raison ». Le service recevabilité qui traite sa demande au Défenseur des droits reprend la même idée en faisant état de «la perte» de son emploi et en lui demandant de fournir son contrat. Dans le courrier envoyé par la suite par le Pôle privé, deux cases sont cochées parmi les quatre répertoriées : « si vous avez été licencié...» et «si votre réclamation porte sur votre évolution de carrière ». Dans la réponse suivante du réclamant, l'affaire déborde largement le cadre initial, puisqu'il est question de pétition de la part de collègues, d'une tentative de suicide, de l'inspection du travail, du tribunal pénal, des prud'hommes et d'un 
avocat, de Pôle emploi, du père de la réclamante, des gens du Bois de Vincennes et toujours de La Presse et du syndicat «qui m'a convoquée pour me dire qu'il fallait que je laisse ma place à d'autres personnes, que j'avais assez travaillé, alors que la direction de $\mathrm{La}$ Presse avait promis... ». Est-il vraiment question de licenciement ou de carrière? Les choses ne sont pas claires. Un nouvel échange de courrier rend compte du déplacement de la qualification des faits au Défenseur des droits. Dorénavant, il est question d'une réclamation relative « au non-renouvellement» du contrat de travail de $\mathrm{M}^{\mathrm{me}} \mathrm{B}$. Et c'est en effet ce que confirme l'entreprise mise en cause, qui transmet tous les contrats d'usage dont elle a bénéficié. Sans rentrer dans le détail de la suite, notons également que la Directe (direction du ministère du Travail), sollicitée à son tour, considère qu'il faut requalifier ces multiples contrats d'usage en CDI.

Les employeurs cherchent à tirer parti de ces ambiguïtés, comme nous allons le montrer en nous attardant pour finir sur le cas de trois machinistes intermittents, qui sont régulièrement réembauchés dans un festival annuel. Son fonctionnement ayant été perturbé par un mouvement social important, à l'instigation du syndicat soutenu par M. G., celui-ci n'est pas réembauché l'année suivante. Y a-t-il un lien de cause à effet ? S'agit-il d'une discrimination syndicale ? L'affaire est d'abord traitée au Défenseur des droits avant d'être portée aux prud'hommes où elle n'est pas tranchée. Au Défenseur, on considère que la réembauche périodique d'un salarié est suffisante pour asseoir la présomption. Or les 3 intermittents sont dans des situations inégales selon ce point de vue : l'un est réembauché depuis 13 ans, l'autre depuis 4 ans, et M. G. depuis 3 ans. Enjoint de justifier sa décision, l'employeur répond d'emblée par un argument économique: ses besoins en machinistes sont moindres car les productions sont moins lourdes et c'est la raison pour laquelle M. G. n'a pas été réembauché l'année suivante. 
Il en va de même pour les deux autres qui, eux aussi, ont participé au mouvement social et ne sont pas non plus réembauchés. Les arguments de l'employeur sont finalement considérés comme insuffisants pour le Défenseur qui mène l'enquête. D'autres éléments renforcent le soupçon d'un lien entre les décisions de nonrenouvellement et les activités syndicales des intermittents, comme des allusions à une confiance "sérieusement ébranlée » dans un compte rendu, des documents fournis incomplets, l'embauche de neuf nouveaux machinistes, etc. La discussion aux prud'hommes conduit à introduire d'autres arguments. L'avocat de l'employeur affirme que les neuf machinistes ont été embauchés sur des « contrats d'usage de renfort », alors que M. G. avait obtenu des «contrats d'usage longs ». De plus, « il ne s'agit pas de non-renouvellement de contrat au sens du Code du travail mais de non-embauche pour la saison suivante. Or il n'existe aucun droit à embauche future au titre d'un contrat passé ! » (Extrait des conclusions de l'avocat de l'employeur).

Les précisions apportées ont vite fait d'embrouiller l'esprit. Et c'est sans doute là l'objectif de l'avocat qui cherche à montrer que M. G. a été écarté pour raisons professionnelles et non parce qu'il était gréviste. Des non-grévistes n'ont-ils pas aussi été écartés ? À notre connaissance, cette affaire n'est pas encore tranchée.

\section{Conclusion}

L'analyse des plaintes déposées au Défenseur rend compte du sentiment d'injustice qu'éprouvent les personnes dont les trajectoires sont particulièrement heurtées. Ces situations redoublent leurs attentes en matière de stabilité et de statuts protecteurs. Mais leur satisfaction est subordonnée au passage de nouvelles épreuves, comme si l'antériorité des parcours ne suffisait pas à attester des compétences. Et chaque nouvelle épreuve peut être le siège d'un jugement à caractère discriminatoire. 
L'autre aspect mis en évidence dans cet article a trait à la diversité des critères incriminés en ces occasions. Ils ne se limitent pas aux critères du sexe, de l'origine, de la race présumée ou de l'âge, tels que les analyses de la discrimination le mettent souvent en évidence à propos des embauches, mais s'étendent à d'autres caractéristiques. Parmi celles-ci, la santé, les grossesses et le handicap occupent une place non négligeable. Même le critère de l'appartenance syndicale semble pouvoir être activé, comme nous l'avons vu. De tels critères sont plus souvent incriminés lorsqu'il est question d'une inégalité de traitement dans la carrière des individus ou au moment des licenciements. Et une analyse plus systématique donnerait probablement à voir que d'autres critères peuvent être impliqués lorsque les individus enchaînent des contrats de courte durée.

Il semble ainsi que les pratiques discriminatoires et leur diversité augmentent mécaniquement avec l'instabilité des parcours professionnels, tandis que la distinction entre discrimination à l'embauche, dans la carrière et au moment du licenciement perd de sa pertinence.

\section{Bibliographie}

Benghalem H. (2016), « La majorité des embauches en contrats courts se font chez un ancien employeur ", Éclairages, études et analyses, $\mathrm{n}^{\circ} 14$, UNEDIC.

Bossu B. (2014), Les discriminations dans les relations de travail devant les cours d'appel. La réalisation contentieuse d'un droit fondamental, Rapport à la mission de recherche Droit et Justice, $462 \mathrm{p}$.

Bresson M. (2016), «Le travailleur non titulaire dans la fonction publique : une figure emblématique des marges de l'emploi ", Revue Française de Socio-Économie, vol. 17, n², pp. 65-83. 
Bruneau C., Dherbécourt C., Flamand J. et Gilles C. (2016), « Marché du travail : un long chemin vers l'égalité », La note d'analyse de France Stratégie, $\mathrm{n}^{\circ} 42$.

Dumortier T. (2016), «L'appréhension juridictionnelle des discriminations dans la fonction publique», in Gründler T. et Thouvenin J.-M. (dir.), La lutte contre les discriminations à l'épreuve de son effectivité. Les obstacles à la reconnaissance juridique des discriminations, Rapport à la mission de recherche Droit et justice, pp. 349-361.

Fontaine F. et Malherbet F. (2016), « Au-delà de l'emploi et des salaires, les conséquences malheureuses du dualisme du marché du travail », in Fontaine F. et Malherbet F. (dir.), CDD vs CDI : Les effets d'un dualisme contractuel, Presses de Sciences Po, pp. 47-60.

Frouin J.-Y. (2015), « La lutte contre les discriminations et l'emploi », in Colloque 10 ans de droit de la non-discrimination, Défenseur des droits, pp. 37-48.

Gaboriau M. (2019), « Faire travailler sans contrat dans la fonction publique. Gestion quotidienne de l'incertitude et préoccupations morales », Revue française de sociologie, vol. 60-1, pp. 43-69.

Marchal E. (2018), «Les parcours d'embauche au regard des critères de discrimination », Chroniques du travail, $\mathrm{n}^{\circ} 8$, pp. 18-35.

Meziani Y. (2014), « Recruter les descendants de migrants en mairie : quotas invisibles ambivalents et reconnaissance au travail », Revue Migrations Société, ${ }^{\circ}$ 152, p. 9-25.

Milin K. (2018), «CDD, CDI : comment évoluent les embauches et les ruptures depuis 25 ans », Dares analyses, $\mathrm{n}^{\circ} 26$.

Moulin J.-J., Labbe E., Sass C. et Gerbaud L. (2009), «Santé et instabilité professionnelle: résultats issus des centres d'examens de santé de l'assurance maladie », Revue d'épidémiologie et de santé publique, vol. 57, n 3, pp. 141-149. 
Picart C. (2014), «Une rotation de la main-d'œuvre presque quintuplée en 30 ans: plus qu'un essor des formes particulières d'emploi, un profond changement de leur usage », Emploi et salaires édition 2014 de l'INSEE.

Remichi-Meziani Y. (2015), Diversifier l'organigramme? Les dilemmes des mairies de Pessac (France) et d'Amsterdam Nieu West (Pays Bas) face à l'enjeu de la diversité des origines, thèse, Université de Bordeaux. 


\section{Annexe :}

\section{Méthodologie}

Cet article prend appui sur une enquête réalisée au Défenseur des droits, où nous avons fait un recensement exhaustif de toutes les réclamations pour discrimination à l'embauche, enregistrées entre 2013 et 2015 : soit 844 réclamations concernant l'accès à l'emploi public ou privé. Partant de cette base exhaustive, nous avons tiré au sort une centaine de plaintes en opérant un codage de leurs principales caractéristiques.

Dans un deuxième temps, nous avons opéré une analyse approfondie des 36 dossiers (parmi les 100) qui ont débouché sur des enquêtes auprès d'employeurs, en explorant les documents fournis et les argumentaires de toutes les parties prenantes: réclamants, mis en cause (les employeurs ou leurs représentants) et juristes du Défenseur. Cette démarche a été complétée par l'exploration des documents produits par le Défenseur, par des entretiens avec 10 juristes chargés de l'instruction de ces dossiers et par l'observation de situations de travail collectif. 
« IL SEMBLE AINSI QUE LES PRATIQUES DISCRIMINATOIRES ET LEUR DIVERSITÉ AUGMENTENT MÉCANIQUEMENT AVEC

L'INSTABILITÉ DES PARCOURS PROFESSIONNELS, TANDIS QUE LA DISTINCTION ENTRE DISCRIMINATION À L'EMBAUCHE, DANS LA CARRIÈRE ET AU MOMENT DU LICENCIEMENT PERD DE SA PERTINENCE ».

Emmanuelle Marchal 